\title{
Study on Second Design of Old Jeans
}

\section{Jinlong Yuan ${ }^{1,2, a}$, Haiyan Fang ${ }^{3, b}$, Yu Huang ${ }^{1, c}$, Qiongfang Zhang ${ }^{1, d}$}

${ }^{1}$ College of Light-Textile Engineering and Art, Anhui Agricultural University, Hefei, Anhui, 230036

${ }^{2}$ Academy of Arts \& Design, Tsinghua University, Beijing, 100084

${ }^{3}$ Anhui Business Vocational College, Hefei, Anhui, 230041

a email, ${ }^{\text {b email, }}{ }^{\mathrm{c}}$ email, ${ }^{\mathrm{d}}$ email

\section{Keywords: Old Jeans, Second Design, Study}

\begin{abstract}
With the constantly changing fashion, clothing generalized phenomenon of excessive consumption, frequent replacement of clothing, resulting in a large number of garment waste, resource utilization is low, serious pollution of the surrounding environment. Following this, recycling, reuse old clothes has become the focus of public attention. On old jeans, in recovery and after the second design, can give new life, creating a considerable "commercial value, environmental value, use value" for business, consumers, many companies have to seize this designer a business opportunity, committed to the secondary design in old jeans, fashion and promoting sustainable development.
\end{abstract}

\section{Introduction}

Mid-19th century, the advent of jeans in the United States, the prevalence of more than 100 years of history has swept the world, jeans with a variety of features, such as simple, easy-going, naturally, loved by consumers around the world, especially young men and women, jeans everywhere. In the new era, the growing science and technology to people's social life has brought greatly facilitated, but also affect the surrounding environment, a variety of clothing new products have emerged, people are constantly chasing new models, produce large amounts of waste products, jeans too. On old jeans, it still has a use function, combined with its current situation, make the necessary "second design" further extend the life of old jeans, improve resource utilization, to avoid contamination of the surrounding environment, effectively alleviate ambient pressure.

\section{Overview of Jeans}

On jeans, it emerged in the 1920s, the English name "jean", from the French "Genes", is intended to Genoa water husband wore denim trousers, a popular 20th century, the popularity of A garment, with an international element. In 1873, the world's first pair of jeans came in 1933, first there is a clear gender identity jeans came in 1953, in "The Wild One" movie, Marlon Brando plays a wild cowboy image, jeans look swept the globe. In 1996, the jeans appear different expression, for example, embroidery, patchwork. In the 1930s, the increasing popularity of jeans, all kinds of cowboy image have appeared in the popular western films, those "bold, handsome," the cowboy image of a young 20th century had a profound impact. Subsequently, the gradual emergence of jeans in the United States east of the city, has become a deep taste marketplace leisure clothing, daily clothing, and even become a kind of "American culture" phenomenon. Subsequently, a series of changes jeans, World War II, has been spread to every corner of the world, different countries have prevailed an jeans trend, impact on people around the world existing values, aesthetics and so on, they gradually jeans daily life play a key role. Young people continue to wear with jeans, added many new elements, such as, "dynamic" and "rebellion", the prevailing wave cowboy culture, and various "youth culture" integration, such as pop, rock, Bingdi cultural phenomenon appears. In addition, under the action of various forces in the world politics and economy, the different types of cultural trends continue to impact, while denim has become a unique cultural phenomenon, present in every corner of the world, has become a fashion. 


\section{Second Design of Jeans and the Importance of Application}

Evolving with the times, "green" has become the eternal theme of the new era, many colleges and universities, companies have held on "green" design competitions. In the "Challenge Cup" contest, there is a survey on recycling old jeans and the second design, research and the application of the project, a large collection of old jeans on the role of "second design", a strong proponent of recycling natural resources, effectively alleviate Japan is increasing the pressure on the environment, which fits well with the new era of our country, have committed to the project study. On old jeans "second design" and use, it is very important, mainly in those areas. First, it avoided a serious waste of resources have been, improve resource utilization, reduce new fabrics appropriate to reduce production costs and improve operational efficiency. Secondly, it helps to reduce emissions of waste, avoiding the surrounding environment has been serious pollution, ecological impacts. Finally, you can promote public awareness of the importance of environmental protection, change the existing dressing lifestyle, it has become an important advocate of energy conservation, focusing on low-carbon life. At the same time, for a designer, we need to continue to expand the design perspective, prompting the design work with colorful art and creative materials, greater artistic creativity, to promote the works of a more modern design features, has a unique charm.

\section{Second Design Discussion of the Old Jeans}

Standing on the perspective of material, the denim fabric has its particularity. In the second design process, the need for different types of clothing and the necessary comparative analysis, continue to explore the unique design method, to ensure that the old jeans design more scientific and reasonable to obtain optimal use. Standing art and design point of view, the need for a comprehensive analysis of source material, design procedures, continue to explore and study in depth the whole process of creation of the second design jeans, designed to further enhance the practice of secondary waste materials through different channels, it presents in terms of artistic expression. In other words, the need to optimize the use of secondary design techniques, create different types of denim products, making it distinctive, continue to attract public attention, stimulate consumption. On this basis, an objective analysis of the characteristics of old jeans, style, flexible application of a variety of techniques, such as embroidery, friction, stitching, make sure the second design more scientific, reasonable, and further extend the life of old jeans to give optimal use, it has a new aesthetic experience, wear with form. You can also optimize the use of relevant techniques, such as material mix, cut, old jeans to make the necessary design derivatives, such as interior decoration, clothing accessories, designed to promote old cowboy second after loading into a simple production process , yet popular fashion element, can walk freely between the two, so that the new jeans to show the works of art can be a form of multi-dimensional show in the world, prompting the old jeans to show their full potential, has a unique charm and more social market potential.

In fabric recycling, for example, since our accession to the WTO, garment consumption concept has undergone a qualitative change in clothing production, design new demands have been put forward, more focus on "second design." On old jeans, in the second design process, often we will optimize the use of fabric recycling methods, such as, hollow, embroidery subsidies, prompting jeans has a new and diverse styles. First, hollow. On hollow, the need under the old denim fabric characteristics, appropriate treatment, such as Artex, dig, transform the fabric itself, may be appropriate to change the way the hollow, forms, etc., fully demonstrates the characteristics of old jeans.

Secondly, embroidery. On embroidery, the need for comprehensive analysis of old jeans existing design intent, optimize the use of colorful embroidery threads, the different types of acupuncture, subtly embroidered, transformation pattern corresponding to achieve different types of decorative effect, prompting old jeans to be used again, to better show their value. Finally, subsidies embroidery. It is called up cutwork, cleverly cut and paste other types of fabric or embroidery sewn onto the old jeans, make it look more "live wave, elegant", creating a gentle beauty, thus in stark contrast produce visual the conflict, to achieve unexpected results. In addition, you can also use the 
sequin embroidery. In the old jeans embroidered on the appropriate prefix related materials, such as beads, sequins flash, achieve brilliant luster effect, so that the old jeans look more elegant, gorgeous, more eye-catching. In the second design, the need to combine the characteristics of old jeans, shape, surface structure appropriate transformation beads, etc., cleverly combined into a special decorative pattern, achieve a better three-dimensional effect, has prompted an elegant old jeans decorative style.

\section{Case Explanation}

On lace design applications, proper leg cut jeans, sewing lace at the edges, this is one of the second design engineering methods, with a distinct "simple, practical" features. This is because this is very difficult to design a secondary low, simple operation, most people can easily complete their own preferences, it has better wear results. Typically, most of the color of dark jeans, funky, lace color choices will be more extensive.

Rivet design applications, based on the old jeans style features, cleverly integrated into the popular punk elements, the jeans in place to add the right amount of rivet, since the rivet with a strong hard sense, it is possible and old denim fabric has the soft feeling the impact of each other, in stark contrast to this ancient bronze, old and pale washed blue color, texture of the old cowboy coupled with special properties, so that after the transformation of the visual device can engender conflict, with distinct Bitterness, time sense and demonstrates the characteristics of jeans.

Remove color in the design process, the need to utilize a liquid disinfectant and other cured with good color effects, reasonable transformation, design jeans, this second design method is also relatively low degree of difficulty, it is possible to achieve a better effect of reconstruction. In the second design process, the appropriate color soak easily remove the site can also be used smear method, to completely remove the original color, after washing, drying process, so having a good old jeans washing effect, hanging with effect. In addition, according to the specific circumstances of old jeans, to develop a viable secondary design, you can use a suitable paint to cover those stains difficult to remove, even old jeans necessary dyeing process, the old jeans were local embroidery, subsidies and other treatment, but also to promote the old jeans into use, full of their own value.

On damaged designs, in conjunction with current fashion trends, skillfully handling the edge of old jeans, partial wear, etc., cleverly integrated into the "retro fashion" element, so that after the second design, and other types of after the dress with ingenious, it will exhibit different characteristics, no less popular jeans latest. Further, in the second design process, the need for flexible application of seemingly superfluous corners material to make a pencil case, bag, can also be made into a unique style of decoration, such as bracelet and necklace pendant.

In jeans, for example, the open-wire technology is mainly reflected in two aspects, namely suturing of open wire, decorative open wire.

\section{Conclusion}

All in all, in our case, belong to the textile industry, the production and consumption country, denim clothing in the clothing market, covering a very wide consumer groups, especially jeans, from children to the elderly into different consumer segments. On old jeans, the "second design" is very necessary, which is a kind of "low-carbon, environmentally friendly" new forms of life, is the theme of the times and the new era coincides through the "second design" after old jeans with a unique style, and does not own the material characteristics jeans conflict can be "fashion, the classic" two seemingly unrelated elements of integration, by the different consumer sectors alike. In addition, old jeans "second design" help to conserve natural resources, appropriate to reduce the pollution of the surrounding environment, to achieve "economic, social and ecological" three benefits of unity, but also conducive to vigorously promote China's "resource-saving and environment-friendly type "social construct, and constantly promote the sustained development of recycling economy, the long-term path to development. 


\section{Acknowledgements}

Anhui Provincial Quality Project (Project Number: 2014jyxm090); Anhui Agricultural University, prosperity and development of philosophy and social science fund project (project number: 2014zs06); Anhui Agriculture University student innovation fund project (Project No. xj2014348)

Anhui Agriculture University student innovation fund project (Project No. xj2014361)

\section{References}

[1] Ji Fengqin, Meng Lu. Secondary Design and Utilization used jeans [J]. Light Industry Science and Technology, 2015, v.31; No.19704: $100+130$.

[2] Hu Weidan. Old jeans "second design" research [D]. Jiangnan University, 2014.

[3] Zhang Zhimin. Application Research [D]. "cowboy" element in popular clothing in Hebei University of Science and Technology, 2013.

[4] Zhang Zhongqi. Secondary Design of Jeans [J]. International Textile Leader, 2010, v.38; No.22903: 67-68 + 70 .

[5] Li Hongqin. Jeans feasibility Jianping secondary usage explore [J] Progress in Textile Science and Technology, 2011, No.15204:. 65-68. 